Neue Elemente und Ephemeride des Cometen 1890... (Brooks März 19).

Aus den Beobachtungen Cambridge Mass. März 21, Paris und Bordeaux April 3, Wien April I 8 wurden unter Berücksichtigung der Parallaxe, Aberration und der Sonnenbreiten folgende Elemente erhalten:

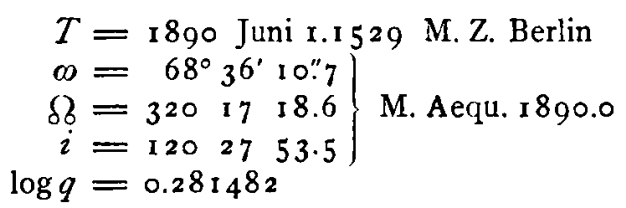

Mittl. Beobachtung $(B-R): \cos \beta \mathrm{d} \lambda=-5.5, \mathrm{~d} \beta=-17.9$

Heliocentrische Aequatorealcoordinaten:

$x=\left[9.925^{2} 29^{\prime} r \cdot \sin \left(v+181^{\circ} 26^{\prime} 20^{\prime \prime} \cdot 2\right)\right.$

$y=[9.960782] r \cdot \sin (v+2883031.9)$

$z=[9.835365] r \cdot \sin \left(z+4^{6} 4738.7\right)$

Ephemeride für $\mathbf{I}^{\text {h }}$ M. Z. Berlin.

\begin{tabular}{|c|c|c|c|c|c|c|}
\hline \multirow{2}{*}{$\frac{1890}{\text { April } 30}$} & \multicolumn{2}{|c|}{$\alpha$ app. } & $\delta$ app. & $\log r$ & $\log \Delta$ & $\mathrm{H}$ \\
\hline & $2 I^{h}$ & $0^{m} 51^{s}$ & $+29^{\circ} 10^{\prime} 6$ & & & \\
\hline Mai I & 20 & 5945 & 2958.4 & & & \\
\hline 2 & & $5^{8} 3^{6}$ & 3047.0 & 0.2894 & $0.275 \mathrm{I}$ & 2.39 \\
\hline 3 & & 5724 & 3 I 36.4 & & & \\
\hline 4 & & $5^{6} 7$ & $32 \quad 26.7$ & & & \\
\hline 5 & & $544^{6}$ & $33 \quad 17.8$ & & & \\
\hline 6 & & $.532 \mathrm{I}$ & $34 \quad 9.6$ & 0.2874 & $0.259^{6}$ & 2.60 \\
\hline 7 & & $5 I 5 I$ & $35 \quad 2.1$ & & & \\
\hline 8 & & $50 \quad 14$ & $35 \quad 55.4$ & & • & \\
\hline 9 & & $48 \quad 3 I$ & $3^{6} \quad 49.4$ & & & \\
\hline 10 & & $46 \quad 4 I$ & $3744 . I$ & 0.2857 & 0.2449 & 2.80 \\
\hline II & & $44 \quad 45$ & $\begin{array}{ll}3^{8} & 39.4\end{array}$ & & & \\
\hline I 2 & & 4241 & 3935.2 & & & \\
\hline 13 & & $40 \quad 29$ & 403 I. 6 & & & \\
\hline 14 & & $3^{8} \quad 9$ & $4 I \quad 28.5$ & 0.2843 & 0.23 I 4 & 3.00 \\
\hline 15 & 20 & 3540 & +4225.9 & & & \\
\hline
\end{tabular}

\begin{tabular}{|c|c|c|c|c|c|c|c|c|c|}
\hline \multicolumn{2}{|c|}{1890} & \multicolumn{3}{|c|}{$\alpha$ app. } & \multicolumn{2}{|c|}{$\delta$ app. } & $\log r$ & $\log 4$ & $\mathbf{H}$ \\
\hline Mai & 15 & $20^{h}$ & $35^{\mathrm{m}}$ & $40^{5}$ & $+42^{\circ}$ & $25 \cdot 9$ & & & \\
\hline & I 6 & & 33 & I & 43 & 23.7 & & & \\
\hline & I 7 & & 30 & 12 & 44 & $2 \times .8$ & & & \\
\hline & 18 & & 27 & 13 & 45 & 20.2 & 0.2832 & 0.2195 & 3.18 \\
\hline & 19 & & 24 & 3 & $4^{6}$ & 188 & & & \\
\hline & 20 & & 20 & 40 & 47 & 17.5 & & & \\
\hline & $2 \mathrm{I}$ & & 17 & 5 & $4^{8}$ & 16.2 & & & \\
\hline & 22 & & 13 & I 6 & 49 & 14.9 & 0.2824 & 0.2096 & $3 \cdot 34$ \\
\hline & 23 & & & 13 & 50 & 13.4 & & & \\
\hline & 24 & & 4 & 541 & $5^{I}$ & II. 5 & & & \\
\hline & 25 & 20 & 01 & 19 & $5^{2}$ & 9.1 & & & \\
\hline & 26 & 19 & 552 & 28 & 53 & 6.2 & 0.2818 & 0.2022 & 3.47 \\
\hline & 27 & & 501 & I 9 & 54 & 2.6 & & & \\
\hline & 28 & & 445 & 50 & 54 & $5^{8.1}$ & & & \\
\hline & 29 & & 39 & 2 & 55 & $5^{2.6}$ & & & \\
\hline & 30 & 19 & 325 & 54 & +56 & 46.1 & 0.2815 & 0.1976 & 3.55 \\
\hline
\end{tabular}

Währing 1890 April 22.

Friedrich Bidschof.

\title{
Entdeckung von zwei Planeten.
}

Am 26. April Nachmittags erhielt die Centralstelle folgendes Telegramm von der Sternwarte in Wien:

Zwei Planeten Palisa 1890 April $2512^{\mathrm{h}} 3^{\mathrm{m}} \mathrm{I}$ M. Z. Wien AR. app. $=25^{\circ} 16^{\circ} 55^{\prime \prime}$ PD. app. $=101^{\circ} 6^{\prime} 29^{\prime \prime}$ tägl. Bew. in AR. : - $15^{\prime}$, in PD.: $-5^{\circ}$. Grösse $13^{\mathrm{m}}$.

I 890 April $2512^{\mathrm{h}} 13^{\mathrm{m}} \cdot 9$ M. Z. Wien AR. app. $=214^{\circ} 54^{\prime} 30^{\prime \prime}$ PD. app. $=100^{\circ} 40^{\prime} \mathrm{I}^{\prime \prime}$ tägl. Bew. in AR. : - I $7^{\prime}$, in PD.: + I'. Grösse $\mathbf{I ~}^{\mathrm{m}}{ }^{\mathrm{m}}$. Wahrscheinlich Scylla.

Weiss.

Das Telegramm konnte wegen einer nothwendigen Rlickfrage erst gegen Mitternacht weiter befördert werden.

Am 27. April Nachmittags telegraphirte Herr Perrotin aus Nizza:

Les deux planètes Palisa ont été trouvées indépendamment cette nuit, avril 26, par Charlois. Voici les observations : $\begin{aligned} \text { Avril } 26 & \text { I }^{\mathrm{h}} 53^{\mathrm{m}} \cdot 5 \text { t. m. Nice AR. app. } & =214^{\circ} 35^{\prime} 53^{\prime \prime} & \text { DP. app. } & =100^{\circ} 40^{\prime} 52^{\prime \prime} \\ 26 & \text { I } 523.2 & =2145916 & =101 & 044^{2}\end{aligned}$

Perrotiv.

Verkauf's-Anzeigen.

Mein 6zölliger Refractor, Objectiv von Reinfelder \& Hertel (Winnecke's Bahnsucher), Montirung von Sendtner (verstellbare Polhöhe), ist billig zu verkaufen.

Dr. Max Wolf, Heidelberg.

$\mathrm{Zu}$ verkaufen ein ausgezeichneter $3^{1 / 2}$ zölliger Schröder'scher Cometensucher mit Doppel-Kreismikrometer. Dr. Oppenheim, Berlin, Blumeshof.

Inhalt zu Nr. 2966. G.V. Schiaparelli. Note sull' aspetto fisico della grande Cometa r882 II. 225. - B. Matthiessen. Beobachtungen der Mondränder und Mondsterne sowie des Kraters Mösting A am Passageninstrument der Karlsruher Sternwarte. 235. - F. Franz. Ephemeride für den Krater Mösting A für die Culmination in Greenwich. 237. - F. Bidschof. Neue Elemente und Ephemeride des Cometen r8go... (Brooks März 19). 239. - Entdeckung von zwei Planeten. 239. - Verkaufs-Anzeigen. 239. 